\title{
SOQPSK-TG STC : a new decoding scheme for a higher bit rate and a better link budget
}

\author{
Alain Thomas, Julien Renaudin, Gregory Blanc, Rami Othman \\ 1 Safran Data Systems 5 Avenue des Andes, 91940 Les Ulis France, \\ alain-dominique.thomas@safrangroup.com
}

\begin{abstract}
To protect telemetry from antenna masking during flight tests, two antennae must be used on-board. To avoid phase opposition of the two transmitted signals at reception, frequency diversity has been used for decades. However with spectrum auctions this technique is no longer compatible with the requirement of higher bit rate. To overcome this issue, well-known modulation format like COFDM may be used at the expense of power efficiency, with few constraints on antennae position. IRIG-106 standard proposed also SOQPSK-TG STC which is efficient both in power and in spectrum at the expense of the antennae position. To demodulate this format a dual-trellis solution has been proposed based on XTCQM representation [1] with results very close to SISO as far as distance between antennae does not exceed half bit delay. Therefore this decoding scheme is a hard decision scheme and consequently is less power efficient when a FEC such as LDPC is used. In this paper a new decoding scheme will be presented with soft decision outputs to increase power efficiency, and with a better tolerance to differential delay.
\end{abstract}

Key words: Flight test, spatial diversity, Space time coding, telemetry.

\section{Introduction}

Transmitting real-time telemetry with low latency on aeronautical channel is very demanding. The data link has to be error free on at least four different channel models from high Doppler spread take-off fading channel to low SNR far flight channel [1]. Spectrum auctions constrain even more the data link pushing it to $C$ band and requiring spectral efficiency to transmit up to $20 \mathrm{Mbps}$ to meet market demand. A first waveform based on COFDM meeting all these requirements has been proposed [1][2] to transmit telemetry from commercial aircraft. However for small aircraft or for carriers with little power available, the amplifier back-off required by COFDM could be incompatible with mission requirements. For such missions SOQPSK-TG combined with equalization techniques can meet power and spectral efficiency on channel with moderate frequency selectivity [3]. However under certain aircraft to ground station geometries, antenna masking occurs, leading to link outage. A MISO hard bit scheme Alamouti like has been proposed [4] to mitigate the shadowing and the associated differential delay based on XTCQM decomposition of STCSOQPSK-TG waveform. In this paper a new low resource decoder with soft output and its performances will be presented.

\section{IRIG-106 STC-SOQPSK-TG}

IRIG-106 has standardized constant envelope waveforms with ever increasing spectral efficiency enabling use of power amplifier at saturation

\begin{tabular}{|c|c|c|c|c|}
\hline Characteristic & $\begin{array}{l}\text { PCM/FM with } \\
\text { single symbol } \\
\text { detection }\end{array}$ & $\begin{array}{c}\text { PCM/FM with } \\
\text { multi-symbol } \\
\text { detection }\end{array}$ & $\begin{array}{c}\text { FQPSK-B, } \\
\text { FQPSK-JR, } \\
\text { SOQPSK-TG }\end{array}$ & ARTM CPM \\
\hline Occupied Bandwidth & 1.16 bit rate & 1.16 bit rate & 0.78 bit rate & 0.56 bit rate \\
\hline $\begin{array}{c}\text { Sensitivity } \\
\left(E_{b} / N_{0} \text { for BEP=1e-5) }\right.\end{array}$ & $11.8-15+\mathrm{dB}$ & $9.5 \mathrm{~dB}$ & $11.8-12.2 \mathrm{~dB}$ & $12.5 \mathrm{~dB}$ \\
\hline Synchronization time & $\begin{array}{l}100 \text { to } 10,000 \\
\text { bits }\end{array}$ & 250 bits & $\begin{array}{l}5,000 \text { to } 30,000 \\
\text { bits }\end{array}$ & $\begin{array}{c}30,000 \text { to } \\
150,000 \text { bits }\end{array}$ \\
\hline $\begin{array}{l}\text { Synchronization } \\
\text { threshold level }\left(\mathrm{E}_{\mathrm{b}} / \mathrm{N}_{0}\right)\end{array}$ & 3 to $4 \mathrm{~dB}$ & $2 \mathrm{~dB}$ & 4.5 to $5 \mathrm{~dB}$ & $8.5 \mathrm{~dB}$ \\
\hline $\begin{array}{c}\text { Phase noise } \\
\text { susceptibility* }\end{array}$ & 2 & 1 & 3 & 4 \\
\hline $\begin{array}{c}\text { Co-channel } \\
\text { interference } \\
\text { susceptibility* }\end{array}$ & 2 & 1 & 3 & 4 \\
\hline
\end{tabular}

Fig. 1 IRIG-106 waveforms comparison

However to prevent data link from on-board antenna masking outage, dual antennae (MISO) transmission is required to ensure errorfree telemetry transmission, and SOQPSK-TG features an interesting trade-off between spectral efficiency with an occupied bandwidth of $0.78^{*}$ bit rate and power efficiency thanks to its $4 \mathrm{~dB}$ synchronization level and its performance when coupled to an ARJA LDPC FEC [5]. Combining SOQPSK-TG and MISO space diversity is however not straightforward as modu- 
lation is not linear and antenna spacing onboard induced a differential delay at reception which is not compatible with Alamouti scheme. In [6] an Alamouti like scheme has been proposed, and a SOQPSK-TG space time decoder based on XTCQM representation given. This scheme has been standardized by IRIG-106 group [7] enabling telemetry transmission with a high quality of service whatever the aircraft to ground station geometry.

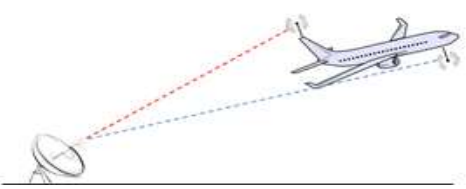

Fig.2a Miso scheme with differential delay

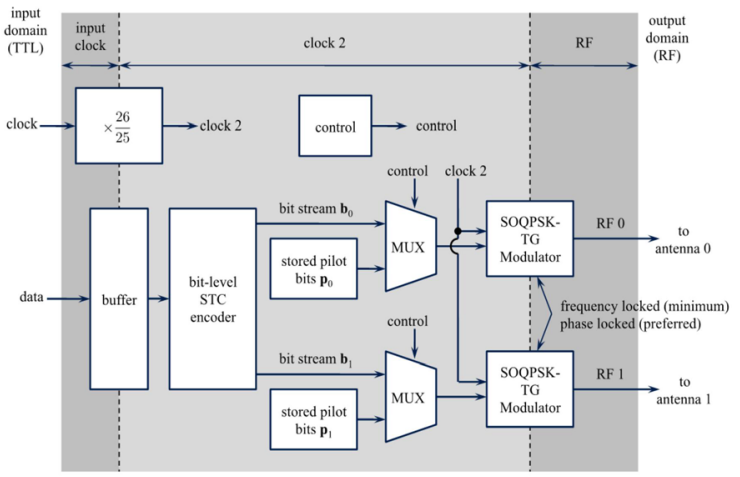

Fig.2b STC on-board modulator

\begin{tabular}{ccccc} 
& \multicolumn{2}{c}{ Alamouti Code } & \multicolumn{3}{c}{ IRIG 106-15 } \\
\hline & \multicolumn{3}{c}{ symbol index } & \multicolumn{2}{c}{ symbol index } \\
& $2 k$ & $2 k+1$ & $2 k$ & $2 k+1$ \\
\hline TX 0 & $s_{2 k}$ & $-s_{2 k+1}^{*}$ & $b_{4 k}, b_{4 k+1}$ & $\bar{b}_{4 k+2}, b_{4 k+3}$ \\
TX 1 & $s_{2 k+1}$ & $s_{2 k}^{*}$ & $b_{4 k+2}, b_{4 k+3}$ & $b_{4 k}, \bar{b}_{4 k+1}$ \\
\hline
\end{tabular}

Fig.2c Bit level STC encoder

As can been noticed on fig. 3 the tolerated differential delay is strictly limited to one bit, and a noticeable BER degradation occurs at half a bit period. Taken into account plane geometry and constraints on antennae location, a direct consequence of this limitation is a limitation of the telemetry rate which cannot be higher than

$$
R \max <\frac{c}{2 \text { Dant }}
$$

Where Dant is the distance between antennae, considering differential delay due to on-board cables has been perfectly compensated.

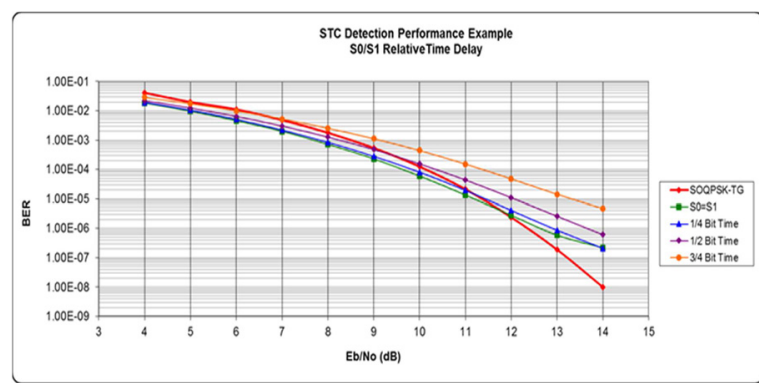

Fig. 3 STC detection performance vs differential time delay

Consequently, the ability to decrease degradation related to differential delay could be a way to relax constraints on antennae position or to increase telemetry rate with the same position constraints. Another constraint of the differential delay on the XTCQM LS decoder [4][6] is it requires to implement two trellis, which increases its complexity.

\section{STC PAM LS decoder}

In [9] a new PAM decomposition was proposed by Othman, STC signal can then be written as

$s_{p}(t) \approx \sum_{i} \rho_{0,2 i}^{p} w_{0}(t-2 i T)-\rho_{1,2 i+1}^{p} w_{1}(t-2 i T-T)+$ $\left(\sum_{i} \rho_{0,2 i+1}^{p} w_{0}(t-2 i T-T)-\rho_{0,2 i}^{p} w_{1}(t-2 i T)\right)$

\section{Where :}

$p \in\{0,1\}$ is the on-board antenna number

$\rho_{0, i}^{p}=\left\{\begin{array}{c}\left(2 b_{i}^{(p)}-1\right) \text { i even } \\ j\left(2 b_{i}^{(p)}-1\right) \text { i odd }\end{array}\right.$

$\rho_{1, i}^{p}=\left\{\begin{array}{c}-j\left(2 b_{i-2}^{(p)}-1\right)\left(2 b_{i-1}^{(p)}-1\right)\left(2 b_{i}^{(p)}-1\right) \mathrm{i} \text { even } \\ -\left(2 b_{i-2}^{(p)}-1\right)\left(2 b_{i-1}^{(p)}-1\right)\left(2 b_{i}^{(p)}-1\right) \mathrm{i} \text { odd }\end{array}\right.$

$b_{i}^{(0)}$ and $b_{i}^{(1)}$ are ith bits feeding respectively channel 0 and 1 encoded accordingly to fig2.C

$w_{0}(t)$ and $w_{1}(t)$ are shaping pulse given in [9] and fig. 4

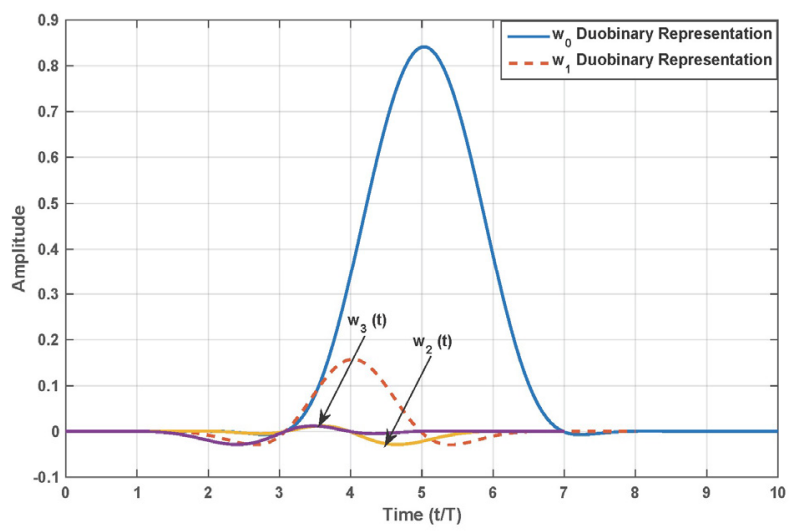

Fig.4 DBD Pulse shape 
On ground antenna I the signal received is

$r_{I}(t)=\left[h_{0, I} s_{0}\left(t-\Delta t_{0, I}\right)+h_{1, I} s_{1}\left(t-\Delta t_{1, I}\right)\right] e^{j 2 \pi \Delta f_{I} t}$
$+z_{I}(t)$

(3)

\section{Where}

$h_{p, I}$ is the LOS channel complex gain between antenna $\mathrm{p}$ and antenna I

$\Delta t_{p, I}$ is propagation delay between antenna $\mathrm{p}$ and antenna $I$ and $\Delta \tau_{I}=\Delta t_{1, I}-\Delta t_{0, I}$

$\Delta f_{I}$ frequency offset, $z_{I}(t)$ additive noise

In Miso scheme $\mathrm{I}=0$, considering perfect carrier synchronization and combining (2) and (3) received signal after ADC could be written

$$
\begin{aligned}
& r_{0}(n) \\
& \approx h_{0} \underbrace{\left[\sum_{i} \rho_{0, i}^{0} w_{0}\left(n T^{\prime}-i T\right)+\sum_{i, i} \rho_{1, i}^{0} w_{1}\left(n T^{\prime}-i T\right)\right]}_{s_{0}\left(n T^{\prime}\right)} \\
& +h_{1} \underbrace{\left[\sum_{i} \rho_{0, i}^{1} w_{0}\left(n T^{\prime}-i T-\Delta \tau\right)+\sum_{i} \rho_{1, i}^{1} w_{1}\left(n T^{\prime}-i T-\Delta \tau\right)\right]}_{s_{1}\left(n T^{\prime}-\Delta \tau\right)} \\
& +z\left(n T^{\prime}\right)
\end{aligned}
$$

(4)

Where $T^{\prime}$ is sampling time. As shown on fig.4 shaping pulse lasts more than $\mathrm{T}$, consequently intersymbol interference remains and has to be filtered. Optimal detection filter is discussed in [10], after filtering and sampling of two TX channels samples can be written using (4) accordingly to Fig.5:

$$
\begin{array}{r}
y(4 k) \approx h_{0} \sum_{i=-1}^{1} \rho_{0,4 k-i}^{0} \widetilde{w}_{0}(i T)+h_{0} \rho_{1,4 k}^{0} \widetilde{w}_{1}(0) \\
+h_{1} \sum_{i=-1}^{1} \rho_{0,4 k-i}^{1} \widetilde{w}_{0}(i T-\Delta \varepsilon T) \\
+h_{1} \rho_{1,4 k}^{1} \widetilde{w}_{1}(-\Delta \varepsilon T)+\tilde{n}(4 k T)
\end{array}
$$

(5)

$$
\begin{aligned}
y_{\Delta \tau}(4 k) \approx h_{0} \sum_{i=-1}^{1} & \rho_{0,4 k-i}^{0} \widetilde{w}_{0}(i T+\Delta \varepsilon T) \\
& +h_{0} \rho_{1,4 k}^{0} \widetilde{w}_{1}(\Delta \varepsilon T) \\
& +h_{1} \sum_{i=-1}^{1} \rho_{0,4 k-i}^{1} \widetilde{w}_{0}(i T) \\
& +h_{1} \rho_{1,4 k}^{1} \widetilde{w}_{1}(0)+\tilde{n}(4 k T+\Delta \varepsilon T)
\end{aligned}
$$

(6)

Where $\widetilde{w}_{i}$ is filtered pulse shape, $\tilde{n}$ is $z$ filtered, and $\Delta \varepsilon$ is nearest integer from $\Delta \tau / T$

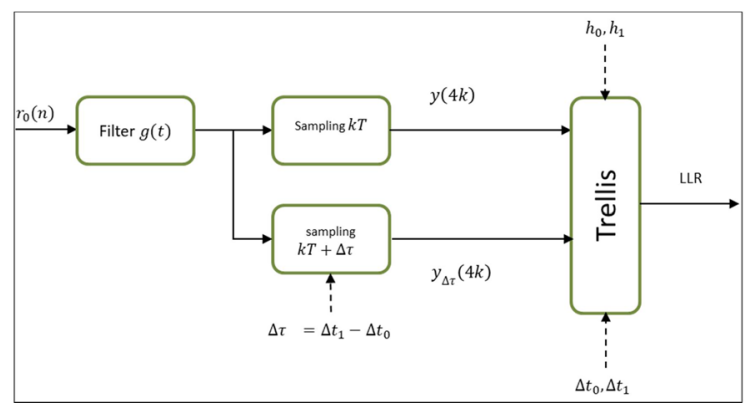

Fig.5 STC SOQPSK-TG decoder

Then the following likelihood function is considered

$\Lambda(\underline{S})=\sum_{n=0}^{(N-1) / 4}\left(\sum_{m=-1}^{2}\left[\left|B_{m, n}^{(0)}\right|^{2}+\left|B_{m, n}^{(\Delta \tau)}\right|^{2}\right]\right)$

(7)

\section{Where}

$B_{m, n}^{(0)}=y(4 n+m)-h_{0}\left(\sum_{i=-1}^{1} \rho_{0,4 n+m-i}^{0} \widetilde{w}_{0}(i T)+\right.$ $\left.\rho_{1,4 n+m}^{0} \widetilde{w}_{1}(0)\right)-h_{1}\left(\sum_{i=-1}^{1} \rho_{0,4 n+m-i}^{1} \widetilde{w}_{0}(i T-\Delta \varepsilon T)+\right.$ $\left.\rho_{1,4 n+m}^{1} \widetilde{w}_{1}(-\Delta \varepsilon T)\right)$

(8)

$$
\begin{aligned}
& B_{m, n}^{(\Delta \tau)}=y_{\Delta \tau}(4 n+m)-h_{0}\left(\sum_{i=-1}^{1} \rho_{0,4 n+m-i}^{0} \widetilde{w}_{0}(i T+\right. \\
& \left.\Delta \varepsilon T)+\rho_{1,4 n+m}^{0} \widetilde{w}_{1}(\Delta \varepsilon T)\right)- \\
& h_{1}\left(\sum_{i=-1}^{1} \rho_{0,4 n+m-i}^{1} \widetilde{w}_{0}(i T)+\rho_{1,4 n+m}^{1} \widetilde{w}_{1}(0)\right)
\end{aligned}
$$

(9)

The ML estimate is then

$\underline{\hat{S}}=\underset{S}{\operatorname{argmin}} \Lambda(\underline{S})$

(10)

Where $S_{n}=\left[\begin{array}{llll}b_{4 n} & b_{4 n+1} & b_{4 n+2} & b_{4 n+3}\end{array}\right]$

Thus the more likely underlying LLR sequence can be estimated with SOVA algorithm using a 16 states trellis (Fig. 5 \& 6)

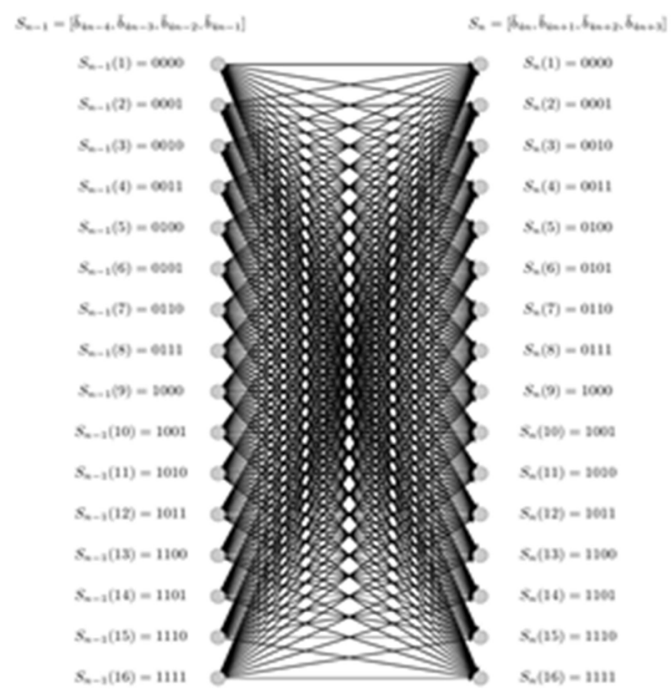

Fig.6 STC-SOQPSK-TG DBD trellis 
Instead of trellises derived from XTCQM decomposition [4], it should be noticed that the expressions (8) and (9) remains unchanged whatever the sign of the differential delay between channels, consequently a unique trellis has to be implemented in the decoder instead of two. Moreover considering (8) and (9) and taken into account that each STC symbol is a block of 4 bits, 2048 sub-metric should be computed to decode at bit level, however developing (8) and (9) as a function of the branch bits leads to consider only 320 sub-metric [10] against 480 in [4]. It could also be noticed that on AWGN channel at high SNR regime DBD PAM decoder outperforms XTCQM decoder of more than $1 \mathrm{~dB}$ at BER $10 \mathrm{E}-6$ for a 0.4 differential delay.

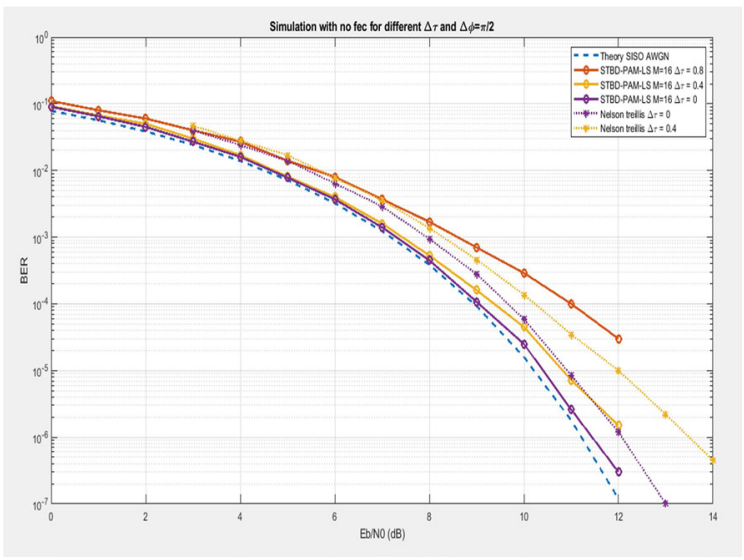

Fig.6 BER proposed decoder vs XTCQM decoder

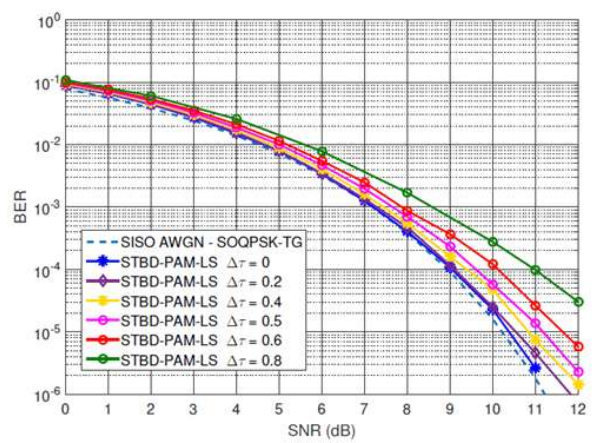

Fig.7 Proposed decoder BER vs differential delay

Instead of improving link budget of $1 \mathrm{~dB}$, looking at Fig6. and 7, as proposed decoder has the same BER performance at $10 \mathrm{E}-6$ for a 0.6 differential delay than the XTCQM decoder at 0.4 differential delay, antennae position constraints could be relaxed of $50 \%$, or bit rate could be increased of $50 \%$ with the same antennae position constraint .

\section{STC PAM LS soft decision decoder}

Soft decisions may be extracted thanks to MAX-log-MAP equivalent SOVA for non binary codes [12]. The classical approach to estimate the underlying bit sequence and its soft information consists then in making a full trellis search up to a certain depth $\delta$ and then performing a trace-back loop, which means, for the proposed trellis, to calculate 256 metrics and to store $256 \delta$ reliability values. Instead of extending all states from one symbol time to the next, a suboptimal technique in [11] consists in extending only the $M$ best states, which is called M-algorithm. Othman in [8] proposed to adapt it to its non-binary STC 4 bits, which leads to calculate 16.M branch metrics.

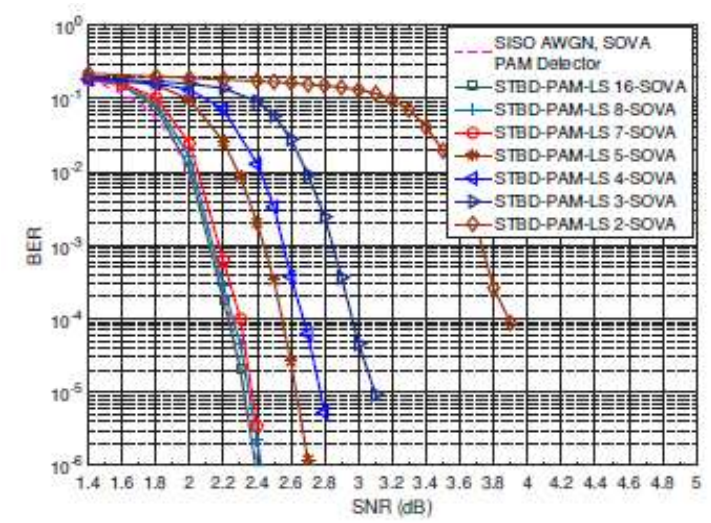

Fig. 8 STC M-algorithm AR4JA BER vs M parameter

To estimate optimal $M$ value decoder has then been simulated on AWGN channel in combination with IRIG-106 AR4JA LDPC code $\mathrm{K}=2 / 3$ $\mathrm{L}=4096$. As shown on Fig. 8 for $\mathrm{M}>4$ BER degradation is below $0.2 \mathrm{~dB}$ dividing by 3 metrics to be calculated, for $M=8$ metrics calculation is divided by 2 at no BER cost. Taken into account Fig. 9 giving required resources, for $M=8$ we thus get a significant gain in gates between the XTCQM hard bit decoder and the proposed one with LLR output.

\begin{tabular}{|c|c|c|c|c|}
\hline & 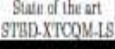 & 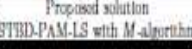 & 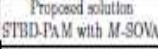 & 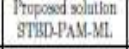 \\
\hline Addition & 3968 & $600+1289$ & $644+176 M$ & 576 \\
\hline Malitpllowiton & getiol & 610 & 64AI & 64 \\
\hline$\pi^{2}$ & 48ता & 221 & .927 & - \\
\hline Conpartsin & 24] & ISM & $46 \mathrm{M}+0$ & 256 \\
\hline $\begin{array}{l}\text { Sorlunp, opernitan a } \\
16 \text { dimmeris }\end{array}$ & 0 & 1 & 1 & II \\
\hline $\begin{array}{l}\text { Nurbir of } \\
\text { sticend wlus }\end{array}$ & 16 & 16 & $17 \mathrm{M}$ & 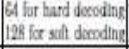 \\
\hline
\end{tabular}

Fig. 9 Operations required by STC decoder

Both decoders have then been simulated using IRIG-106 AR4JA FEC on Fig. 10 exhibiting a $1.5 \mathrm{~dB}$ gain. Once again this gain could be used to relax link budget or antenna position constraint or increase bit rate as the proposed decoder enables a higher differential delay margin at constant BER. 


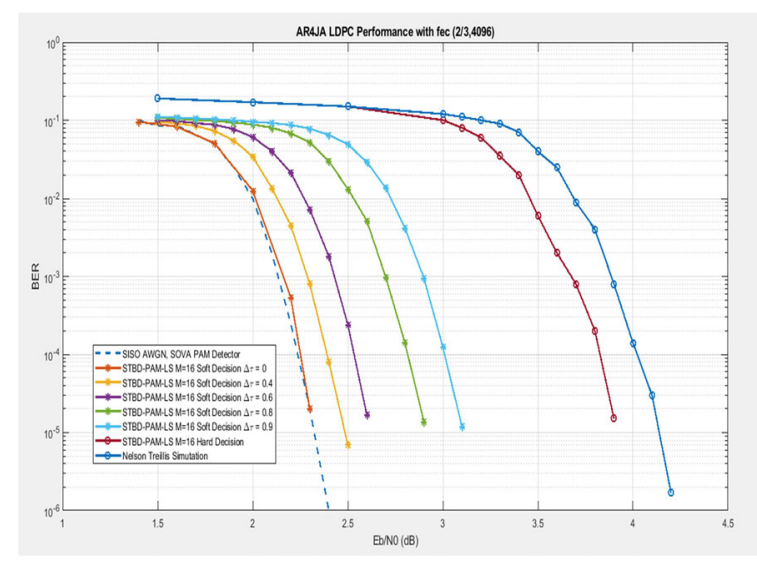

Fig.10 Decoders performance with AR4JA 2/3,4096

\section{Conclusion}

Space time coding applied on SOQPSK-TG telemetry link, when constant envelope modulation is mandatory because of on-board power constraint, is a very performant solution to solve the antenna masking problem using a MISO scheme. However the telemetry bit rate is upper-bounded by a maximum bit rate that depends on differential delay (1). XTCQM decoder thus enables, with no significant loss, a differential delay of a quarter of bit, which means for a $20 \mathrm{Mbps}$ link a maximum distance of $3.75 \mathrm{~m}$ between antennae with a perfect calibration of path from modulator outputs to antennae inputs. With proposed PAM-LS decoder it should be possible with the same antennae location to reach $32 \mathrm{Mbps}$ at equivalent $\mathrm{Eb} / \mathrm{NO}$ and $40 \mathrm{Mbps}$ at the cost of an extra $0.2 \mathrm{~dB}$. Moreover using IRIG-106 LDPC FEC PAM-LS decoder enables to gain an extra $1.5 \mathrm{~dB}$ link budget thanks to soft decision output at the price of reduced gate consumption.

\section{References}

[1] A. Skrzypczak, A. Thomas, G. Duponchel, "Paradigms optimization for a C-band COFDM telemetry with high bit efficiency", International telemetry proceedings, Las Vegas, NV, Oct 2013

[2] L. Falga "The challenging development and the entry into service of C-Band telemetry at Airbus Test centre", ETTC proceedings, Nov 2014

[3] A. Cole-Rhodes, H. Umuolo, F.Moazzami "Realtime CMA equalization for SOQPSK for aeronautical telemetry", International telemetry proceedings, San Diego, CA, Oct 2014

[4] M. Rice, T. Nelson, J. Palmer, C. Lavin, K. Temple "Space-Time coding for telemetry : part II Decoder and system performance", IEEE Transactions and Electronic Systems, vol. PP, pp. 1-1, Aug 2017

[5] K. Temple "Telemetry standards that improve link availability", ETTC proceedings, $2018 \mathrm{DOI}$ 10.5162/ettc2018/2.2

[6] N.T Nelson "Space time coding with offset modulations", PhD thesis Brigham Youth University, Provo 2007

[7] Secretariat, Range Commander Council "Space time coding", RCC document IRIG106-17 Appen$\operatorname{dix} 2 \mathrm{E}$

[8] R. Othman "Etude de techniques de reception des modulations de fréquence pour la télémesure aéronautique",PhD thesis tel-02430542 2019

[9] R. Othman, A. Skrzypczak, Y. Louët, "PAM Decomposition of Ternary CPM with Duobinary Encoding", IEEE Transactions on Communications, vol. 65, no. 10, pp. 4274-4284, Oct. 2017

[10] Geoghegan, Mark, "Optimal Linear Detection of SOQPSK," in International Telemetering Conference Proceedings, Oct. 2002

[11] K.K.Y Wong "The soft output M-algorithm and its application", Queen's University, 2006

[12] J.Tan, GL. Stuber "A MAP equivalent Sova for non binary Turbo Codes", ICC 2000, Conference Record, vol2 pp602-606, June 2000 